\title{
On the Solutions of Three-Dimensional Rational Difference Equation Systems
}

\author{
H. S. Alayachi, ${ }^{1,2}$ A. Q. Khan $\mathbb{D}^{3}{ }^{3}$ and M. S. M. Noorani ${ }^{1}$ \\ ${ }^{1}$ School of Mathematical Sciences, Faculty of Science and Technology, Universiti Kebangsaan Malaysia, Bangi, Selangor, Malaysia \\ ${ }^{2}$ Department of Mathematics, College of Science, Taibah University, Al-Madinah, Saudi Arabia \\ ${ }^{3}$ Department of Mathematics, University of Azad Jammu and Kashmir, Muzaffarabad 13100, Pakistan
}

Correspondence should be addressed to A. Q. Khan; abdulqadeerkhan1@gmail.com

Received 26 July 2020; Revised 28 March 2021; Accepted 29 March 2021; Published 16 April 2021

Academic Editor: Ljubisa Kocinac

Copyright ( $\odot 2021 \mathrm{H}$. S. Alayachi et al. This is an open access article distributed under the Creative Commons Attribution License, which permits unrestricted use, distribution, and reproduction in any medium, provided the original work is properly cited.

In this paper, we are interested in a technique for solving some nonlinear rational systems of difference equations of third order, in three-dimensional case as a special case of the following system: $x_{n+1}=\left(y_{n} z_{n-1} / y_{n} \pm x_{n-2}\right), y_{n+1}=\left(z_{n} x_{n-1} / z_{n} \pm y_{n-2}\right)$, and $z_{n+1}=$ $\left(x_{n} y_{n-1} / x_{n} \pm z_{n-2}\right)$ with initial conditions $x_{-2}, x_{-1}, x_{0}, y_{-2}, y_{-1}, y_{0}, z_{-2}, z_{-1}$, and $z_{0}$ are nonzero real numbers. Moreover, we study some behavior of the systems such as the boundedness of solutions for such systems. Finally, we present some numerical examples by giving some numerical values for the initial values of each case. Some figures have been given to explain the behavior of the obtained solutions in the case of numerical examples by using the mathematical program MATLAB to confirm the obtained results.

\section{Introduction}

We believe that difference equations, also referred to as recursive sequence, are a hot topic here as there has been increasing interest in the study of qualitative analysis of difference equations and systems of difference equations. Difference equations appear naturally as discrete analogues and as numerical solutions of differential and delay differential equations having applications in biology, ecology, economics, physics, computer sciences, and so on. Although difference equations are very simple in form, it is extremely difficult to understand thoroughly the behaviors of their solution (see [1-9] and the references cited therein). Recently, a great effort has been made in studying the qualitative analysis of rational difference equations and rational difference system (see [10-35]).

The study of the nonlinear rational difference equations is quite challenging and rewarding $[2,8]$. The results about these equations offer prototypes towards the development of the basic theory of the global behavior of nonlinear difference equations [1]. Recently, many researchers have investigated the behavior of the solution of difference equations. Difference equations arise in the situations in which the discrete values of the independent variable involve. Many practical phenomena are modeled with the help of difference equations [1]. In engineering, difference equations arise in control engineering, digital signal processing, electrical networks, etc. In social sciences, difference equations arise to study the national income of a country and then its variation with time, Cobweb phenomenon in economics, etc.

There are many papers related to the difference equation system, for example, the periodicity of the positive solutions of the rational difference equations system:

$$
\begin{aligned}
& x_{n+1}=\frac{1}{z_{n}}, \\
& y_{n+1}=\frac{y_{n}}{x_{n-1} y_{n-1}}, \\
& z_{n+1}=\frac{1}{x_{n-1}},
\end{aligned}
$$

has been obtained by Cinar in [4]. 
Khan et al. [6] studied the equilibrium points, local asymptotic stability of an equilibrium point, instability of equilibrium points, periodicity behavior of positive solutions, and global character of an equilibrium point of a fourth-order system of rational difference equations of the form:

$$
\begin{aligned}
& x_{n+1}=\frac{\alpha x_{n-3}}{\beta+\gamma y_{n} y_{n-1} y_{n-2} y_{n-3}}, \\
& y_{n+1}=\frac{\alpha_{1} y_{n-3}}{\beta_{1}+\gamma_{1} x_{n} x_{n-1} x_{n-2} x_{n-3}} .
\end{aligned}
$$

Elabbasy et al. [7] has obtained the solution of particular cases of the following general system of difference equations:

$$
\begin{aligned}
& x_{n+1}=\frac{a_{1}+a_{2} y_{n}}{a_{3} z_{n}+a_{4} x_{n-1} z_{n}}, \\
& y_{n+1}=\frac{b_{1} z_{n-1}+b_{2} z_{n}}{b_{3} x_{n} y_{n}+b_{4} x_{n} y_{n-1}}, \\
& z_{n+1}=\frac{c_{1} z_{n-1}+c_{2} z_{n}}{c_{3} x_{n-1} y_{n-1}+c_{4} x_{n-1} y_{n}+c_{5} x_{n} y_{n}} .
\end{aligned}
$$

In [36], Elsayed et al. dealt with the solutions of the systems of the difference equations:

$$
\begin{aligned}
& x_{n+1}=\frac{1}{x_{n-p} y_{n-p}}, \\
& y_{n+1}=\frac{x_{n-p} y_{n-p}}{x_{n-q} y_{n-q}}, \\
& x_{n+1}=\frac{1}{x_{n-p} y_{n-p} z_{n-p}}, \\
& y_{n+1}=\frac{x_{n-p} y_{n-p} z_{n-p}}{x_{n-q} y_{n-q} z_{n-q}}, \\
& z_{n+1}=\frac{x_{n-q} y_{n-q} z_{n-q}}{x_{n-r} y_{n-r} z_{n-r}} .
\end{aligned}
$$

Kurbanli [13-15] investigated the behavior of the solutions of the difference equation systems:

$$
\begin{aligned}
& x_{n+1}=\frac{x_{n-1}}{x_{n-1} y_{n}-1}, \\
& y_{n+1}=\frac{y_{n-1}}{y_{n-1} x_{n}-1}, \\
& z_{n+1}=\frac{1}{z_{n} y_{n}}, \\
& x_{n+1}=\frac{x_{n-1}}{x_{n-1} y_{n}-1}, \\
& y_{n+1}=\frac{y_{n-1}}{y_{n-1} x_{n}-1}, \\
& z_{n+1}=\frac{z_{n-1}}{z_{n-1} y_{n}-1}, \\
& x_{n+1}=\frac{x_{n-1}}{x_{n-1} y_{n}-1}, \\
& y_{n+1}=\frac{y_{n-1}}{y_{n-1} x_{n}-1}, \\
& z_{n+1}=\frac{x_{n}}{z_{n-1} y_{n}} .
\end{aligned}
$$

In [21], Yalcinkaya et al. studied the periodic character of the following two systems of difference equations:

$$
\begin{aligned}
& x_{n+1}^{(1)}=\frac{x_{n}^{(2)}}{x_{n}^{(2)}-1}, x_{n+1}^{(2)}=\frac{x_{n}^{(3)}}{x_{n}^{(3)}-1}, \ldots, x_{n+1}^{(k)}=\frac{x_{n}^{(1)}}{x_{n}^{(1)}-1}, \\
& x_{n+1}^{(1)}=\frac{x_{n}^{(k)}}{x_{n}^{(k)}-1}, x_{n+1}^{(2)}=\frac{x_{n}^{(1)}}{x_{n}^{(1)}-1}, \ldots, x_{n+1}^{(k)}=\frac{x_{n}^{(k-1)}}{x_{n}^{(k-1)}-1},
\end{aligned}
$$

where the initial values are nonzero real numbers for $x_{0}^{(1)}, x_{0}^{(2)}, \ldots, x_{0}^{(k)} \neq 1$.

In [37], Zhang et al. studied the boundedness, the persistence, and global asymptotic stability of the positive solutions of the system of difference equations:

$$
\begin{aligned}
& x_{n}=A+\frac{1}{y_{n-p}}, \\
& y_{n}=A+\frac{y_{n-1}}{x_{n-r} y_{n-s}} .
\end{aligned}
$$

Zkan and Kurbanli [38] have investigated the periodical solutions of the following system of third-order rational difference equations: 


$$
\begin{aligned}
& x_{n+1}=\frac{y_{n-2}}{-1 \pm y_{n-2} x_{n-1} y_{n}}, \\
& y_{n+1}=\frac{x_{n-2}}{-1 \pm x_{n-2} y_{n-1} x_{n}}, \\
& z_{n+1}=\frac{x_{n-2}+y_{n-2}}{-1 \pm x_{n-2} y_{n-1} x_{n}} .
\end{aligned}
$$

Similar to the references above, this paper is devoted to obtain the form of the solutions of the following third-order systems of rational difference equations:

$$
\begin{aligned}
& x_{n+1}=\frac{y_{n} z_{n-1}}{y_{n} \pm x_{n-2}}, \\
& y_{n+1}=\frac{z_{n} x_{n-1}}{z_{n} \pm y_{n-2}}, \\
& z_{n+1}=\frac{x_{n} y_{n-1}}{x_{n} \pm z_{n-2}},
\end{aligned}
$$

where the initial conditions $x_{-2}, x_{-1}, x_{0}, y_{-2}$, $y_{-1}, y_{0}, z_{-2}, z_{-1}$, and $z_{0}$ are nonzero real numbers.

\section{The System:}

$$
\begin{aligned}
& x_{n+1}==\left(y_{n} z_{n-1} / y_{n}+x_{n-2}\right), y_{n+1}==\left(z_{n} x_{n-1} /\right. \\
& \left.z_{n}+y_{n-2}\right), \text { and } z_{n+1}==\left(x_{n} y_{n-1} / x_{n}+z_{n-2}\right)
\end{aligned}
$$

We obtain the form of solutions of the following system of difference equations:

$$
\begin{aligned}
& x_{n+1}=\frac{y_{n} z_{n-1}}{y_{n}+x_{n-2}}, \\
& y_{n+1}=\frac{z_{n} x_{n-1}}{z_{n}+y_{n-2}}, \\
& z_{n+1}=\frac{x_{n} y_{n-1}}{x_{n}+z_{n-2}} .
\end{aligned}
$$

Theorem 1. If $\left\{x_{n}, y_{n}, z_{n}\right\}$ be the solution of (10), then

$$
\begin{aligned}
& x_{6 n-2}=\frac{x_{-2} y_{0}^{3 n}}{\prod_{i=0}^{n-1}\left(x_{-2}+(6 i) y_{0}\right)\left(x_{-2}+(6 i+2)\right) y_{0}\left(x_{-2}+(6 i+4) y_{0}\right)}, \\
& x_{6 n-1}=\frac{x_{-1} z_{0}^{3 n}}{\prod_{i=0}^{n-1}\left(y_{-2}+(6 i+1) z_{0}\right)\left(y_{-2}+(6 i+3) z_{0}\right)\left(y_{-2}+(6 i+5) z_{0}\right)}, \\
& x_{6 n}=\frac{x_{0}^{3 n+1}}{\prod_{i=0}^{n-1}\left(z_{-2}+(6 i+2) x_{0}\right)\left(z_{-2}+(6 i+4) x_{0}\right)\left(z_{-2}+(6 i+6) x_{0}\right)}, \\
& x_{6 n+1}=\frac{z_{-1} y_{0}^{3 n+1}}{\left(x_{-2}+y_{0}\right) \prod_{i=0}^{n-1}\left(x_{-2}+(6 i+3) y_{0}\right)\left(x_{-2}+(6 i+5) y_{0}\right)\left(x_{-2}+(6 i+7) y_{0}\right)} \text {, } \\
& x_{6 n+2}=\frac{z_{0}^{3 n+2}}{\left(y_{-2}+2 z_{0}\right) \prod_{i=0}^{n-1}\left(y_{-2}+(6 i+4) z_{0}\right)\left(y_{-2}+(6 i+6) z_{0}\right)\left(y_{-2}+(6 i+8) z_{0}\right)}, \\
& x_{6 n+3}=\frac{y_{-1} x_{0}^{3 n+2}}{\left(z_{-2}+x_{0}\right)\left(z_{-2}+3 x_{0}\right) \prod_{i=0}^{n-1}\left(z_{-2}+(6 i+5) x_{0}\right)\left(z_{-2}+(6 i+7) x_{0}\right)\left(z_{-2}+(6 i+9) x_{0}\right)}, \\
& y_{6 n-2}=\frac{y_{-2} z_{0}^{3 n}}{\prod_{i=0}^{n-1}\left(y_{-2}+(6 i) z_{0}\right)\left(y_{-2}+(6 i+2) z_{0}\right)\left(y_{-2}+(6 i+4) z_{0}\right)}, \\
& y_{6 n-1}=\frac{y_{-1} x_{0}^{3 n}}{\prod_{i=0}^{n-1}\left(z_{-2}+(6 i+1) x_{0}\right)\left(z_{-2}+(6 i+3) x_{0}\right)\left(z_{-2}+(6 i+5) x_{0}\right)}, \\
& y_{6 n}=\frac{y_{0}^{3 n+1}}{\prod_{i=0}^{n-1}\left(x_{-2}+(6 i+2) y_{0}\right)\left(x_{-2}+(6 i+4) y_{0}\right)\left(x_{-2}+(6 i+6) y_{0}\right)}, \\
& y_{6 n+1}=\frac{z_{-1} z_{0}^{3 n+1}}{\left(y_{-2}+z_{0}\right) \prod_{i=0}^{n-1}\left(y_{-2}+(6 i+3) z_{0}\right)\left(y_{-2}+(6 i+5) z_{0}\right)\left(y_{-2}+(6 i+7) z_{0}\right)}, \\
& y_{6 n+2}=\frac{x_{0}^{3 n+2}}{\left(z_{-2}+2 x_{0}\right) \prod_{i=0}^{n-1}\left(z_{-2}+(6 i+4) x_{0}\right)\left(z_{-2}+(6 i+6) x_{0}\right)\left(z_{-2}+(6 i+8) x_{0}\right)} \text {, }
\end{aligned}
$$




$$
\begin{aligned}
y_{6 n+3} & =\frac{z_{-1} y_{0}^{3 n+2}}{\left(x_{-2}+y_{0}\right)\left(x_{-2}+3 y_{0}\right) \prod_{i=0}^{n-1}\left(x_{-2}+(6 i+5) y_{0}\right)\left(x_{-2}+(6 i+7) y_{0}\right)\left(x_{-2}+(6 i+9) y_{0}\right)}, \\
z_{6 n-2} & =\frac{z_{-2} x_{0}^{3 n}}{\prod_{i=0}^{n-1}\left(z_{-2}+(6 i) x_{0}\right)\left(z_{-2}+(6 i+2) x_{0}\right)\left(z_{-2}+(6 i+4) x_{0}\right)}, \\
z_{6 n-1} & =\frac{z_{-1} y_{0}^{3 n}}{\prod_{i=0}^{n-1}\left(x_{-2}+(6 i+1) y_{0}\right)\left(x_{-2}+(6 i+3) y_{0}\right)\left(x_{-2}+(6 i+5) y_{0}\right)}, \\
z_{6 n} & =\frac{z_{0}^{3 n+1}}{\prod_{i=0}^{n-1}\left(y_{-2}+(6 i+2) z_{0}\right)\left(y_{-2}+(6 i+4) z_{0}\right)\left(y_{-2}+(6 i+6) z_{0}\right)}, \\
z_{6 n+1} & =\frac{y_{-1} x_{0}^{3 n+1}}{\left(z_{-2}+x_{0}\right) \prod_{i=0}^{n-1}\left(z_{-2}+(6 i+3) x_{0}\right)\left(z_{-2}+(6 i+5) x_{0}\right)\left(z_{-2}+(6 i+7) x_{0}\right)}, \\
z_{6 n+2} & =\frac{y_{0}^{3 n+2}}{\left(x_{-2}+2 y_{0}\right) \prod_{i=0}^{n-1}\left(x_{-2}+(6 i+4) y_{0}\right)\left(x_{-2}+(6 i+6) y_{0}\right)\left(x_{-2}+(6 i+8) y_{0}\right)}, \\
z_{6 n+3} & =\frac{x_{-1} z_{0}^{3 n+2}}{\left(y_{-2}+z_{0}\right)\left(y_{-2}+3 z_{0}\right) \prod_{i=0}^{n-1}\left(y_{-2}+(6 i+5) z_{0}\right)\left(y_{-2}+(6 i+7) z_{0}\right)\left(y_{-2}+(6 i+9) z_{0}\right)} .
\end{aligned}
$$

Proof. Obviously, results are true for $n=0$. Suppose that it is also true for $n-1$, i.e.,

$$
\begin{aligned}
& x_{6 n-8}=\frac{x_{-2} y_{0}^{3 n-3}}{\left.\prod_{i=0}^{n-2}\left(x_{-2}+(6 i) y_{0}\right)\left(x_{-2}+(6 i+2)\right) y_{0}\right)\left(x_{-2}+(6 i+4) y_{0}\right)}, \\
& x_{6 n-7}=\frac{x_{-1} z_{0}^{3 n-3}}{\prod_{i=0}^{n-2}\left(y_{-2}+(6 i+1) z_{0}\right)\left(y_{-2}+(6 i+3) z_{0}\right)\left(y_{-2}+(6 i+5) z_{0}\right)}, \\
& x_{6 n-6}=\frac{x_{0}^{3 n-2}}{\prod_{i=0}^{n-2}\left(z_{-2}+(6 i+2) x_{0}\right)\left(z_{-2}+(6 i+4) x_{0}\right)\left(z_{-2}+(6 i+6) x_{0}\right)}, \\
& x_{6 n-5}=\frac{z_{-1} y_{0}^{3 n-2}}{\left(x_{-2}+y_{0}\right) \prod_{i=0}^{n-2}\left(x_{-2}+(6 i+3) y_{0}\right)\left(x_{-2}+(6 i+5) y_{0}\right)\left(x_{-2}+(6 i+7) y_{0}\right)} \text {, } \\
& x_{6 n-4}=\frac{z_{0}^{3 n-1}}{\left(y_{-2}+2 z_{0}\right) \prod_{i=0}^{n-2}\left(y_{-2}+(6 i+4) z_{0}\right)\left(y_{-2}+(6 i+6) z_{0}\right)\left(y_{-2}+(6 i+8) z_{0}\right)}, \\
& x_{6 n-3}=\frac{y_{-1} x_{0}^{3 n-1}}{\left(z_{-2}+x_{0}\right)\left(z_{-2}+3 x_{0}\right) \prod_{i=0}^{n-2}\left(z_{-2}+(6 i+5) x_{0}\right)\left(z_{-2}+(6 i+7) x_{0}\right)\left(z_{-2}+(6 i+9) x_{0}\right)}, \\
& y_{6 n-8}=\frac{y_{-2} z_{0}^{3 n-3}}{\prod_{i=0}^{n-2}\left(y_{-2}+(6 i) z_{0}\right)\left(y_{-2}+(6 i+2) z_{0}\right)\left(y_{-2}+(6 i+4) z_{0}\right)}, \\
& y_{6 n-7}=\frac{y_{-1} x_{0}^{3 n-3}}{\prod_{i=0}^{n-2}\left(z_{-2}+(6 i+1) x_{0}\right)\left(z_{-2}+(6 i+3) x_{0}\right)\left(z_{-2}+(6 i+5) x_{0}\right)}, \\
& y_{6 n-6}=\frac{y_{0}^{3 n-2}}{\prod_{i=0}^{n-2}\left(x_{-2}+(6 i+2) y_{0}\right)\left(x_{-2}+(6 i+4) y_{0}\right)\left(x_{-2}+(6 i+6) y_{0}\right)},
\end{aligned}
$$




$$
\begin{aligned}
& y_{6 n-5}=\frac{x_{-1} z_{0}^{3 n-2}}{\left(y_{-2}+z_{0}\right) \prod_{i=0}^{n-2}\left(y_{-2}+(6 i+3) z_{0}\right)\left(y_{-2}+(6 i+5) z_{0}\right)\left(y_{-2}+(6 i+7) z_{0}\right)}, \\
& y_{6 n-4}=\frac{x_{0}^{3 n-1}}{\left(z_{-2}+2 x_{0}\right) \prod_{i=0}^{n-2}\left(z_{-2}+(6 i+4) x_{0}\right)\left(z_{-2}+(6 i+6) x_{0}\right)\left(z_{-2}+(6 i+8) x_{0}\right)}, \\
& y_{6 n-3}=\frac{z_{-1} y_{0}^{3 n-1}}{\left(x_{-2}+y_{0}\right)\left(x_{-2}+3 y_{0}\right) \prod_{i=0}^{n-2}\left(x_{-2}+(6 i+5) y_{0}\right)\left(x_{-2}+(6 i+7) y_{0}\right)\left(x_{-2}+(6 i+9) y_{0}\right)}, \\
& z_{6 n-8}=\frac{z_{-2} x_{0}^{3 n-3}}{\prod_{i=0}^{n-2}\left(z_{-2}+(6 i) x_{0}\right)\left(z_{-2}+(6 i+2) x_{0}\right)\left(z_{-2}+(6 i+4) x_{0}\right)}, \\
& z_{6 n-7}=\frac{z_{-1} y_{0}^{3 n-3}}{\prod_{i=0}^{n-2}\left(x_{-2}+(6 i+1) y_{0}\right)\left(x_{-2}+(6 i+3) y_{0}\right)\left(x_{-2}+(6 i+5) y_{0}\right)}, \\
& z_{6 n-6}=\frac{z_{0}^{3 n-2}}{\prod_{i=0}^{n-2}\left(y_{-2}+(6 i+2) z_{0}\right)\left(y_{-2}+(6 i+4) z_{0}\right)\left(y_{-2}+(6 i+6) z_{0}\right)}, \\
& z_{6 n-4}=\frac{y_{-1} x_{0}^{3 n-2}}{\left(z_{-2}+x_{0}\right) \prod_{i=0}^{n-2}\left(z_{-2}+(6 i+3) x_{0}\right)\left(z_{-2}+(6 i+5) x_{0}\right)\left(z_{-2}+(6 i+7) x_{0}\right)}, \\
& z_{6 n-3}=\frac{y_{0}^{3 n-1}}{\left(x_{-2}+2 y_{0}\right) \prod_{i=0}^{n-2}\left(x_{-2}+(6 i+4) y_{0}\right)\left(x_{-2}+(6 i+6) y_{0}\right)\left(x_{-2}+(6 i+8) y_{0}\right)},
\end{aligned}
$$

From equation (10), it follows that

$$
\begin{aligned}
x_{6 n-2}= & \frac{z_{6 n-4} y_{6 n-3}}{y_{6 n-3}+x_{6 n-5}} \\
& \left(y_{0}^{3 n-1} /\left(x_{-2}+2 y_{0}\right) \prod_{i=0}^{n-2}\left(x_{-2}+(6 i+4) y_{0}\right)\left(x_{-2}+(6 i+6) y_{0}\right)\left(x_{-2}+(6 i+8) y_{0}\right)\right) \\
= & \frac{\left(z_{-1} y_{0}^{3 n-1} /\left(x_{-2}+y_{0}\right)\left(x_{-2}+3 y_{0}\right) \prod_{i=0}^{n-2}\left(x_{-2}+(6 i+5) y_{0}\right)\left(x_{-2}+(6 i+7) y_{0}\right)\left(x_{-2}+(6 i+9) y_{0}\right)\right)}{\left(z_{-1} y_{0}^{3 n-1} /\left(x_{-2}+y_{0}\right)\left(x_{-2}+3 y_{0}\right) \prod_{i=0}^{n-2}\left(x_{-2}+(6 i+5) y_{0}\right)\left(x_{-2}+(6 i+7) y_{0}\right)\left(x_{-2}+(6 i+9) y_{0}\right)\right)+} \\
& \frac{\left(z_{-1} y_{0}^{3 n-2} /\left(x_{-2}+y_{0}\right) \prod_{i=0}^{n-2}\left(x_{-2}+(6 i+3) y_{0}\right)\left(x_{-2}+(6 i+5) y_{0}\right)\left(x_{-2}+(6 i+7) y_{0}\right)\right)}{y_{0}+\left(x_{-2}+3 y_{0} \prod_{i=0}^{n-2}\left(x_{-2}+(6 i+9) y_{0}\right) / \prod_{i=0}^{n-2}\left(x_{-2}+(6 i+3) y_{0}\right)\right)} \\
= & \frac{\left(y_{0}^{3 n} /\left(x_{-2}+2 y_{0}\right) \prod_{i=0}^{n-2}\left(x_{-2}+(6 i+4) y_{0}\right)\left(x_{-2}+(6 i+6) y_{0}\right)\left(x_{-2}+(6 i+8) y_{0}\right)\right)}{\left.y_{-2}^{3 n} /\left(x_{-2}+2 y_{0}\right) \prod_{i=0}^{n-2}\left(x_{-2}+(6 i+4) y_{0}\right)\left(x_{-2}+(6 i+6) y_{0}\right)\left(x_{-2}+(6 i+8) y_{0}\right)\right)} \\
= & \frac{1}{\left(x_{-2}+2 y_{0}\right) \prod_{i=0}^{n-2}\left(x_{-2}+(6 i+4) y_{0}\right)\left(x_{-2}+(6 i+6) y_{0}\right)\left(x_{-2}+(6 i+8) y_{0}\right)}\left(\frac{1}{x_{-2}+(6 n-2) y_{0}}\right) .
\end{aligned}
$$


Now, one can see that

$$
x_{6 n-2}=\frac{x_{-2} y_{0}^{3 n}}{\prod_{i=0}^{n-1}\left(x_{-2}+(6 i) y_{0}\right)\left(x_{-2}+(6 i+2) y_{0}\right)\left(x_{-2}+(6 i+4) y_{0}\right)} .
$$

Moreover, from (10), one has

$$
\begin{aligned}
y_{6 n-2}= & \frac{x_{6 n-4} z_{6 n-3}}{z_{6 n-3}+y_{6 n-5}} \\
& \left(z_{0}^{3 n-1} /\left(y_{-2}+2 z_{0}\right) \prod_{i=0}^{n-2}\left(y_{-2}+(6 i+4) z_{0}\right)\left(y_{-2}+(6 i+6) z_{0}\right)\left(y_{-2}+(6 i+8) z_{0}\right)\right) \\
= & \frac{\left(x_{-1} z_{0}^{3 n-1} /\left(y_{-2}+z_{0}\right)\left(y_{-2}+3 z_{0}\right) \prod_{i=0}^{n-2}\left(y_{-2}+(6 i+5) z_{0}\right)\left(y_{-2}+(6 i+7) z_{0}\right)\left(y_{-2}+(6 i+9) z_{0}\right)\right)}{\left(x_{-1} z_{0}^{3 n-1} /\left(y_{-2}+z_{0}\right)\left(y_{-2}+3 z_{0}\right) \prod_{i=0}^{n-2}\left(y_{-2}+(6 i+5) z_{0}\right)\left(y_{-2}+(6 i+7) z_{0}\right)\left(y_{-2}+(6 i+9) z_{0}\right)\right)+} \\
= & \frac{\left(x_{0}^{3 n} z_{0}^{3 n-2} /\left(y_{-2}+z_{0}\right) \prod_{i=0}^{n-2}\left(y_{-2}+(6 i+3) z_{0}\right)\left(y_{-2}+(6 i+5) z_{0}\right)\left(y_{-2}+(6 i+7) z_{0}\right)\right)}{z_{0}+\left(\left(y_{-2}+3 z_{0}\right) \prod_{i=0}^{n-2}\left(y_{-2}+(6 i+9) z_{0}\right) / \prod_{i=0}^{n-2}\left(y_{-2}+(6 i+3) z_{0}\right)\right)} \\
= & \frac{\left(z_{0}^{3 n} /\left(y_{-2}+2 z_{0}\right) \prod_{i=0}^{n-2}\left(y_{-2}+(6 i+4) z_{0}\right)\left(y_{-2}+(6 i+6) z_{0}\right)\left(y_{-2}+(6 i+8) z_{0}\right)\right)}{z_{0}+\left(y_{-2}+(6 n-3) z_{0}\right)} \\
= & \frac{\left(z_{0}^{3 n} /\left(y_{-2}+2 z_{0}\right) \prod_{i=0}^{n-2}\left(y_{-2}+(6 i+4) z_{0}\right)\left(y_{-2}+(6 i+6) z_{0}\right)\left(y_{-2}+(6 i+8) z_{0}\right)\right)}{\left.(6 n-2) z_{0}\right)} .
\end{aligned}
$$

Then,

$$
y_{6 n-2}=\frac{y_{-2} z_{0}^{3 n}}{\left(y_{-2}+2 z_{0}\right) \prod_{i=0}^{n-1}\left(y_{-2}+(6 i) z_{0}\right)\left(y_{-2}+(6 i+2) z_{0}\right)\left(y_{-2}+(6 i+4) z_{0}\right)} .
$$


Finally, from equation (10),

$$
\begin{aligned}
z_{6 n-2}= & \frac{y_{6 n-4} x_{6 n-3}}{x_{6 n-3}+z_{6 n-5}} \\
& \left(x_{0}^{3 n-1} /\left(z_{-2}+2 x_{0}\right) \prod_{i=0}^{n-2}\left(z_{-2}+(6 i+4) x_{0}\right)\left(z_{-2}+(6 i+6) x_{0}\right)\left(z_{-2}+(6 i+8) x_{0}\right)\right) \\
= & \frac{\left(y_{-1} x_{0}^{3 n-1} /\left(z_{-2}+x_{0}\right)\left(z_{-2}+3 x_{0}\right) \prod_{i=0}^{n-2}\left(z_{-2}+(6 i+5) x_{0}\right)\left(z_{-2}+(6 i+7) x_{0}\right)\left(z_{-2}+(6 i+9) x_{0}\right)\right)}{\left(y_{-1} x_{0}^{3 n-1} /\left(z_{-2}+x_{0}\right)\left(z_{-2}+3 x_{0}\right) \prod_{i=0}^{n-2}\left(z_{-2}+(6 i+5) x_{0}\right)\left(z_{-2}+(6 i+7) x_{0}\right)\left(z_{-2}+(6 i+9) x_{0}\right)\right)+} \\
& \left(y_{-1} x_{0}^{3 n-2} /\left(z_{-2}+x_{0}\right) \prod_{i=0}^{n-2}\left(z_{-2}+(6 i+3) x_{0}\right)\left(z_{-2}+(6 i+5) x_{0}\right)\left(z_{-2}+(6 i+7) x_{0}\right)\right) \\
= & \frac{\left(x_{0}^{3 n} /\left(z_{-2}+2 x_{0}\right) \prod_{i=0}^{n-2}\left(z_{-2}+(6 i+4) x_{0}\right)\left(z_{-2}+(6 i+6) x_{0}\right)\left(z_{-2}+(6 i+8) x_{0}\right)\right)}{x_{0}+\left(\left(z_{-2}+3 x_{0}\right) \prod_{i=0}^{n-2}\left(z_{-2}+(6 i+9) x_{0}\right) / \prod_{i=0}^{n-2}\left(z_{-2}+(6 i+3) x_{0}\right)\right)} \\
= & \frac{\left(x_{0}^{3 n} /\left(z_{-2}+2 x_{0}\right) \prod_{i=0}^{n-2}\left(z_{-2}+(6 i+4) x_{0}\right)\left(z_{-2}+(6 i+6) x_{0}\right)\left(z_{-2}+(6 i+8) x_{0}\right)\right)}{x_{0}+\left(z_{-2}+(6 n-3) x_{0}\right)} .
\end{aligned}
$$

Thus,

$$
z_{3 n-2}=\frac{z_{-2} x_{0}^{3 n}}{\prod_{i=0}^{n-1}\left(z_{-2}+(6 i) x_{0}\right)\left(z_{-2}+(6 i+2) x_{0}\right)\left(z_{-2}+(6 i+4) x_{0}\right)} .
$$

In a similar way, other relations can also be proved.

Lemma 1. If $\left\{x_{n}, y_{n}, z_{n}\right\}$ is the +ve solution of (10), then it is bounded as well as converge to zero.

Proof. From (10),

$$
\begin{aligned}
& x_{n+1}=\frac{y_{n} z_{n-1}}{y_{n}+x_{n-2}} \leq z_{n-1}, \\
& y_{n+1}=\frac{z_{n} x_{n-1}}{z_{n}+y_{n-2}} \leq x_{n-1}, \\
& z_{n+1}=\frac{x_{n} y_{n-1}}{x_{n}+z_{n-2}} \leq y_{n-1},
\end{aligned}
$$

we see that

$$
\begin{aligned}
& x_{n+4} \leq z_{n+2}, z_{n+2} \leq y_{n}, y_{n} \leq x_{n-2}, \Rightarrow x_{n+4}<x_{n-2} \\
& y_{n+4} \leq x_{n+2}, x_{n+2} \leq z_{n}, z_{n} \leq y_{n-2}, \Rightarrow y_{n+4}<y_{n-2}, \\
& z_{n+4} \leq y_{n+2}, y_{n+2} \leq x_{n}, x_{n} \leq z_{n-2}, \Rightarrow z_{n+4}<z_{n-2} .
\end{aligned}
$$

Therefore, subsequences $\left\{x_{6 n-2}\right\}_{n=0}^{\infty}, \ldots,\left\{x_{6 n+3}\right\}_{n=0}^{\infty}$ are nonincreasing and so are bounded from above by $M=\max \left\{x_{-2}, x_{-1}, x_{0}, x_{1}, x_{2}, x_{3}\right\}$. Similarly, the subsequences $\left\{y_{6 n-2}\right\}_{n=0}^{\infty}, \ldots,\left\{y_{6 n+3}\right\}_{n=0}^{\infty}$ are nonincreasing and hence bounded above by $M=\max \left\{y_{-2}, y_{-1}, y_{0}, y_{1}, y_{2}, y_{3}\right\}$ and $\left\{z_{6 n-2}\right\}_{n=0}^{\infty}, \ldots,\left\{z_{6 n+3}\right\}_{n=0}^{\infty}$ are nonincreasing and hence bounded from above by $M=\max \left\{z_{-2}, z_{-1}, z_{0}, z_{1}\right.$, $\left.z_{2}, z_{3}\right\}$.

Example 1. Figure 1 represents the dynamics of (10) with $x_{-2}=0.18, x_{-1}=0.15, x_{0}=0.13, y_{-2}=0.26, \quad y_{-1}=0.17$, $y_{0}=-0.13, z_{-2}=0.14, z_{-1}=0.19$, and $z_{0}=0.15$.

\section{The System:}

$$
\begin{aligned}
& x_{n+1}==\left(y_{n} z_{n-1} / y_{n}+x_{n-2}\right), y_{n+1}==\left(z_{n} x_{n-1} /\right. \\
& \left.z_{n}+y_{n-2}\right), \text { and } z_{n+1}==\left(x_{n} y_{n-1} / x_{n}-z_{n-2}\right)
\end{aligned}
$$

Here, we will discuss solutions of the following system:

$$
\begin{aligned}
& x_{n+1}=\frac{y_{n} z_{n-1}}{y_{n}+x_{n-2}}, \\
& y_{n+1}=\frac{z_{n} x_{n-1}}{z_{n}+y_{n-2}}, \\
& z_{n+1}=\frac{x_{n} y_{n-1}}{x_{n}-z_{n-2}},
\end{aligned}
$$

where $n \in \mathbb{N}_{0}$ and nonzero initial conditions such that $x_{-2} \neq \pm y_{0}, x_{-2} \neq 2 y_{0}, y_{-2} \neq \pm z_{0}, y_{-2} \neq-2 z_{0}, z_{-2} \neq x_{0}, z_{-2}$ $\neq 2 x_{0}$, and $z_{-2} \neq 3 x_{0}$.

Theorem 2. If $\left\{x_{n}, y_{n}, z_{n}\right\}$ are solutions of (21), then solutions of (21) are represented by the following formulas for $n=0,1, \ldots$ : 


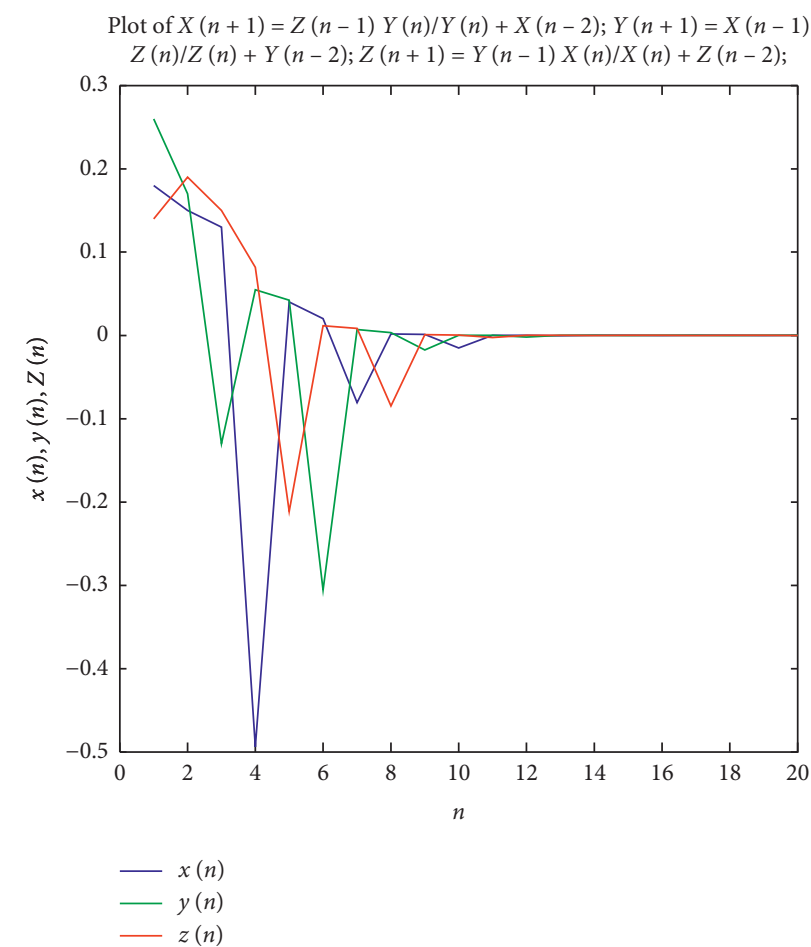

Figure 1: Difference system (10).

$$
\begin{aligned}
x_{6 n-2} & =\frac{y_{0}^{3 n}}{x_{-2}^{2 n-1}\left(x_{-2}-2 y_{0}\right)^{n}}, \\
x_{6 n-1} & =\frac{x_{-1} z_{0}^{3 n}}{\left(y_{-2}+z_{0}\right)^{2 n}\left(y_{-2}-z_{0}\right)^{n}}, \\
x_{6 n} & =\frac{(-1)^{n} x_{0}^{3 n+1}}{z_{-2}^{n}\left(z_{-2}-2 x_{0}\right)^{2 n}}, \\
x_{6 n+1} & =\frac{(-1)^{n} z_{-1} y_{0}^{3 n+1}}{\left(x_{-2}+y_{0}\right)^{n+1}\left(x_{-2}-y_{0}\right)^{2 n}}, \\
x_{6 n+2} & =\frac{(-1)^{n} z_{0}^{3 n+2}}{y_{-2}^{2 n}\left(y_{-2}+2 z_{0}\right)^{n+1}}, \\
x_{6 n+3} & =\frac{y_{-1} x_{0}^{3 n+2}}{\left(z_{-2}-x_{0}\right)^{2 n+1}\left(z_{-2}-3 x_{0}\right)^{n+1}}, \\
y_{6 n} & =\frac{(-1)^{n} z_{0}^{3 n}}{x_{-2}^{2 n}\left(x_{-2}-2 y_{0}\right)^{n}}, \\
y_{6 n-1} & =\frac{x_{-1}^{3 n+1} z_{0}^{3 n+1}}{\left(y_{-2}+z_{0}\right)^{2 n+1}\left(y_{-2}-z_{0}\right)^{n}}, \\
y_{-2}{ }^{2 n-1}\left(y_{-2}+2 z_{0}\right)^{n} & y_{0}^{3 n}
\end{aligned}
$$

$$
\begin{aligned}
y_{6 n+2} & =\frac{(-1)^{n+1} x_{0}^{3 n+2}}{z_{-2}^{n}\left(z_{-2}-2 x_{0}\right)^{2 n+1}}, \\
y_{6 n+3} & =\frac{(-1)^{n+1} z_{-1} y_{0}^{3 n+2}}{\left(x_{-2}-y_{0}\right)^{2 n+1}\left(x_{-2}+y_{0}\right)^{n+1}}, \\
z_{6 n-2} & =\frac{(-1)^{n} x_{0}^{3 n}}{z_{-2}^{n-1}\left(z_{-2}-2 x_{0}\right)^{2 n}}, \\
z_{6 n-1} & =\frac{(-1)^{n} z_{-1} y_{0}^{3 n}}{\left(x_{-2}-y_{0}\right)^{2 n}\left(x_{-2}+y_{0}\right)^{n}}, \\
z_{6 n} & =\frac{(-1)^{n} z_{0}^{3 n+1}}{y_{-2}^{2 n}\left(y_{-2}+2 z_{0}\right)^{n}}, \\
z_{6 n+1} & =\frac{-y_{-1} x_{0}^{3 n+1}}{\left(z_{-2}-x_{0}\right)^{2 n+1}\left(z_{-2}-3 x_{0}\right)^{n}}, \\
z_{6 n+2} & =\frac{-y_{0}^{3 n+2}}{x_{-2}^{2 n+1}\left(x_{-2}-2 y_{0}\right)^{n}}, \\
z_{6 n+3} & =\frac{-x_{-1} z_{0}^{3 n+2}}{\left(y_{-2}-z_{0}\right)^{n}\left(y_{-2}+z_{0}\right)^{2 n+2}} .
\end{aligned}
$$

Proof. Obviously, results are true for $n=0$. Now, one suppose that, for $n-1$, it is also true, i.e., 


$$
\begin{aligned}
& x_{6 n-8}=\frac{y_{0}^{3 n-3}}{x_{-2}^{2 n-3}\left(x_{-2}-2 y_{0}\right)^{n-1}}, \\
& x_{6 n-7}=\frac{x_{-1} z_{0}^{3 n-3}}{\left(y_{-2}+z_{0}\right)^{2 n-2}\left(y_{-2}-z_{0}\right)^{n-1}} \text {, } \\
& x_{6 n-6}=\frac{(-1)^{n-1} x_{0}^{3 n-2}}{z_{-2}^{n-1}\left(z_{-2}-2 x_{0}\right)^{2 n-2}}, \\
& x_{6 n-5}=\frac{(-1)^{n-1} z_{-1} y_{0}^{3 n-2}}{\left(x_{-2}+y_{0}\right)^{n}\left(x_{-2}-y_{0}\right)^{2 n-2}} \text {, } \\
& x_{6 n-4}=\frac{(-1)^{n-1} z_{0}^{3 n-1}}{y_{-2}^{2 n-2}\left(y_{-2}+2 z_{0}\right)^{n}} \\
& x_{6 n-3}=\frac{y_{-1} x_{0}^{3 n-1}}{\left(z_{-2}-x_{0}\right)^{2 n-1}\left(z_{-2}-3 x_{0}\right)^{n}}, \\
& y_{6 n-8}=\frac{(-1)^{n-1} z_{0}^{3 n-3}}{y_{-2}^{2 n-3}\left(y_{-2}+2 z_{0}\right)^{n-1}} \text {, } \\
& y_{6 n-7}=\frac{y_{-1} x_{0}^{3 n-3}}{\left(x_{0}-z_{-2}\right)^{2 n-2}\left(z_{-2}-3 x_{0}\right)^{n-1}} \text {, } \\
& y_{6 n-6}=\frac{y_{0}^{3 n-2}}{x_{-2}^{2 n-2}\left(x_{-2}-2 y_{0}\right)^{n-1}} \text {, } \\
& y_{6 n-5}=\frac{x_{-1} z_{0}^{3 n-2}}{\left(y_{-2}+z_{0}\right)^{2 n-1}\left(y_{-2}-z_{0}\right)^{n-1}} \text {, } \\
& y_{6 n-4}=\frac{(-1)^{n} x_{0}^{3 n-1}}{z_{-2}^{n-1}\left(z_{-2}-2 x_{0}\right)^{2 n-1}} \text {, } \\
& y_{6 n-3}=\frac{(-1)^{n} z_{-1} y_{0}^{3 n-1}}{\left(x_{-2}-y_{0}\right)^{2 n-1}\left(x_{-2}+y_{0}\right)^{n}} \text {, } \\
& z_{6 n-8}=\frac{(-1)^{n-1} x_{0}^{3 n-3}}{z_{-2}^{n-2}\left(z_{-2}-2 x_{0}\right)^{2 n-2}} \text {, } \\
& z_{6 n-7}=\frac{(-1)^{n-1} z_{-1} y_{0}^{3 n-3}}{\left(x_{-2}-y_{0}\right)^{2 n-2}\left(x_{-2}+y_{0}\right)^{n-1}} \text {, } \\
& z_{6 n-6}=\frac{(-1)^{n-1} z_{0}^{3 n-2}}{y_{-2}^{2 n-2}\left(y_{-2}+2 z_{0}\right)^{n-1}} \text {, } \\
& z_{6 n-5}=\frac{-y_{-1} x_{0}^{3 n-2}}{\left(z_{-2}-x_{0}\right)^{2 n-1}\left(z_{-2}-3 x_{0}\right)^{n-1}} \text {, } \\
& z_{6 n-4} \frac{-y_{0}^{3 n-1}}{x_{-2}^{2 n-1}\left(x_{-2}-2 y_{0}\right)^{n-1}} \text {, } \\
& z_{6 n-3}=\frac{-x_{-1} z_{0}^{3 n-1}}{\left(y_{-2}-z_{0}\right)^{n-1}\left(y_{-2}+z_{0}\right)^{2 n}} \text {. }
\end{aligned}
$$


Now, from equation (21), it follows that

$$
\begin{aligned}
x_{6 n-2}= & \frac{z_{6 n-4} y_{6 n-3}}{y_{6 n-3}+x_{6 n-5}} \\
= & \frac{\left(-y_{0}^{3 n-1} / x_{-2}^{2 n-1}\left(x_{-2}-2 y_{0}\right)^{n-1}\right)\left((-1)^{n} z_{-1} y_{0}^{3 n-1} /\left(x_{-2}-y_{0}\right)^{2 n-1}\left(x_{-2}+y_{0}\right)^{n}\right)}{\left((-1)^{n} z_{-1} y_{0}^{3 n-1} /\left(x_{-2}-y_{0}\right)^{2 n-1}\left(x_{-2}+y_{0}\right)^{n}\right)+\left((-1)^{n-1} z_{-1} y_{0}^{3 n-2} /\left(x_{-2}+y_{0}\right)^{n}\left(x_{-2}-y_{0}\right)^{2 n-2}\right)} \\
= & \frac{\left(-y_{0}^{3 n-1} / x_{-2}^{2 n-1}\left(x_{-2}-2 y_{0}\right)^{n-1}\right) y_{0}}{y_{0}-\left(x_{-2}-y_{0}\right)}=\frac{y_{0}^{3 n}}{x_{-2}^{2 n-1}\left(x_{-2}-2 y_{0}\right)^{n}}, \\
y_{6 n-1} & \frac{x_{6 n-3} z_{6 n-2}}{z_{6 n-2}+y_{6 n-4}}=\frac{\left(y_{-1} x_{0}^{3 n-1} /\left(z_{-2}-x_{0}\right)^{2 n-1}\left(z_{-2}-3 x_{0}\right)^{n}\right)\left((-1)^{n} x_{0}^{3 n} / z_{-2}^{n-1}\left(z_{-2}-2 x_{0}\right)^{2 n}\right)}{\left((-1)^{n} x_{0}^{3 n} / z_{-2}^{n-1}\left(z_{-2}-2 x_{0}\right)^{2 n}\right)+\left((-1)^{n} x_{0}^{3 n-1} / z_{-2}^{n-1}\left(z_{-2}-2 x_{0}\right)^{2 n-1}\right)} \\
= & \frac{\left(y_{-1} x_{0}^{3 n-1} /\left(z_{-2}-x_{0}\right)^{2 n-1}\left(z_{-2}-3 x_{0}\right)^{n}\right) x_{0}}{x_{0}+z_{-2}-2 x_{0}}=\frac{y_{-1} x_{0}^{3 n}}{\left(z_{-2}-x_{0}\right)^{2 n}\left(z_{-2}-3 x_{0}\right)^{n}} \\
z_{6 n}= & \frac{y_{6 n-2} x_{6 n-1}}{x_{6 n-1}-z_{6 n-3}}=\frac{\left((-1)^{n} z_{0}^{3 n} / y_{-2}^{2 n-1}\left(y_{-2}+2 z_{0}\right)^{n}\right)\left(x_{-1} z_{0}^{3 n} /\left(y_{-2}+z_{0}\right)^{2 n}\left(y_{-2}-z_{0}\right)^{n}\right)}{\left(x_{-1} z_{0}^{3 n} /\left(y_{-2}+z_{0}\right)^{2 n}\left(y_{-2}-z_{0}\right)^{n}\right)-\left(-x_{-1} z_{0}^{3 n-1} /\left(y_{-2}-z_{0}\right)^{n-1}\left(y_{-2}+z_{0}\right)^{2 n} \%\right)} \\
= & \frac{\left((-1)^{n} z_{0}^{3 n} / y_{-2}^{2 n-1}\left(y_{-2}+2 z_{0}\right)^{n}\right) z_{0}}{z_{0}+y_{-2}-z_{0}}=\frac{(-1)^{n} z_{0}^{3 n+1}}{y_{-2}^{2 n}\left(y_{-2}+2 z_{0}\right)^{n}} .
\end{aligned}
$$

Also, from equation (21), one can see that

$$
\begin{aligned}
x_{6 n+1} & =\frac{z_{6 n-1} y_{6 n}}{y_{6 n}+x_{6 n-2}}=\frac{\left((-1)^{n} z_{-1} y_{0}^{3 n} /\left(x_{-2}-y_{0}\right)^{2 n}\left(x_{-2}+y_{0}\right)^{n}\right)\left(y_{0}^{3 n+1} / x_{-2}^{2 n}\left(x_{-2}-2 y_{0}\right)^{n}\right)}{\left(y_{0}^{3 n+1} / x_{-2}^{2 n}\left(x_{-2}-2 y_{0}\right)^{n}\right)+\left(y_{0}^{3 n} / x_{-2}^{2 n-1}\left(x_{-2}-2 y_{0}\right)^{n}\right)} \\
& =\frac{\left((-1)^{n} z_{-1} y_{0}^{3 n} /\left(x_{-2}-y_{0}\right)^{2 n}\left(x_{-2}+y_{0}\right)^{n}\right) y_{0}}{y_{0}+x_{-2}}=\frac{(-1)^{n} z_{-1} y_{0}^{3 n+1}}{\left(x_{-2}-y_{0}\right)^{2 n}\left(x_{-2}+y_{0}\right)^{n+1}}, \\
y_{6 n+2} & =\frac{x_{6 n} z_{6 n+1}}{z_{6 n+1}+y_{6 n-1}}=\frac{\left((-1)^{n} x_{0}^{3 n+1} / z_{-2}^{n}\left(z_{-2}-2 x_{0}\right)^{2 n}\right)\left(-y_{-1} x_{0}^{3 n+1} /\left(z_{-2}-x_{0}\right)^{2 n+1}\left(z_{-2}-3 x_{0}\right)^{n}\right)}{\left(-y_{-1} x_{0}^{3 n+1} /\left(z_{-2}-x_{0}\right)^{2 n+1}\left(z_{-2}-3 x_{0}\right)^{n}\right)+\left(y_{-1} x_{0}^{3 n} /\left(x_{0}-z_{-2}\right)^{2 n}\left(z_{-2}-3 x_{0}\right)^{n}\right)} \\
& =\frac{\left((-1)^{n} x_{0}^{3 n+1} / z_{-2}^{n}\left(z_{-2}-2 x_{0}\right)^{2 n}\right) x_{0}}{x_{0}-\left(z_{-2}-x_{0}\right)}=\frac{(-1)^{n+1} x_{0}^{3 n+2}}{z_{-2}^{n}\left(z_{-2}-2 x_{0}\right)^{2 n+1}}, \\
z_{6 n+3}= & \frac{y_{6 n+1} x_{6 n+2}}{x_{6 n+2}-z_{6 n}} \\
= & \frac{\left(x_{-1} z_{0}^{3 n+1} /\left(y_{-2}+z_{0}\right)^{2 n+1}\left(y_{-2}-z_{0}\right)^{n}\right) z_{0}}{z_{0}-\left(y_{-2}+2 z_{0}\right)}=\frac{-x_{-1} z_{0}^{3 n+2}}{\left(y_{-2}+z_{0}\right)^{2 n+2}\left(y_{-2}-z_{0}\right)^{n}} .
\end{aligned}
$$

In a similar pattern, one can prove the other relation.

Example 2. Figure 2 represents the dynamics of (21) with $x_{-2}=0.8, x_{-1}=0.5, x_{0}=0.3, \quad y_{-2}=0.26 \quad y_{-1}=0.17, y_{0}=$ $-0.13, z_{-2}=0.14, z_{-1}=0.19$, and $z_{0}=0.15$. 


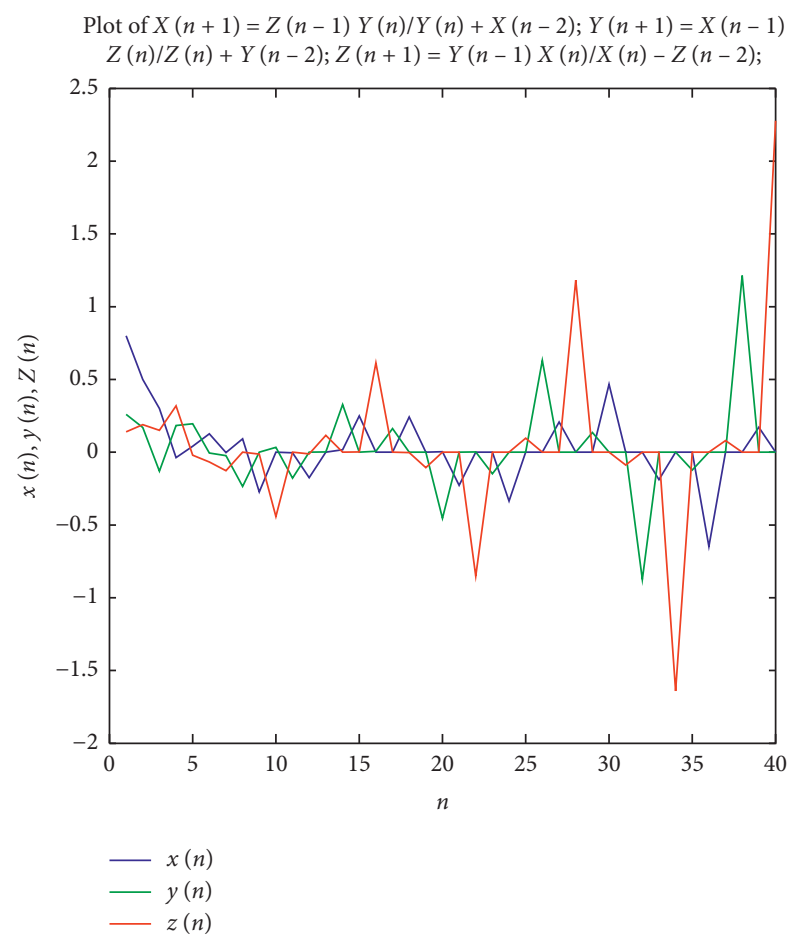

Figure 2: Difference system (21).

In a similar pattern, one can prove the following cases.

4. The System: $x_{n+1}==\left(y_{n} z_{n-1} / y_{n}-x_{n-2}\right), y_{n+1}$ $==\left(z_{n} x_{n-1} / z_{n}-y_{n-2}\right)$, and $z_{n+1}==\left(x_{n} y_{n-1} /\right.$ $\left.x_{n}-z_{n-2}\right)$

We discuss the solutions of the following system:

$$
\begin{aligned}
& x_{n+1}=\frac{y_{n} z_{n-1}}{y_{n}-x_{n-2}}, \\
& y_{n+1}=\frac{z_{n} x_{n-1}}{z_{n}-y_{n-2}}, \\
& z_{n+1}=\frac{x_{n} y_{n-1}}{x_{n}-z_{n-2}},
\end{aligned}
$$

where $n \in \mathbb{N}_{0}$ and nonzero initial conditions such that $x_{-2} \neq y_{0}, y_{-2} \neq z_{0}$, and $x_{0} \neq z_{-2}$.

Theorem 3. If $\left\{x_{n}, y_{n}, z_{n}\right\}$ are solutions of (26), then

$$
\begin{aligned}
x_{6 n-2} & =x_{-2}\left(\frac{y_{0}}{x_{-2}}\right)^{3 n}, \\
x_{6 n-1} & =x_{-1}\left(\frac{z_{0}}{z_{0}-y_{-2}}\right)^{3 n}, \\
x_{6 n} & =x_{0}\left(\frac{x_{0}}{z_{-2}}\right)^{3 n}, \\
x_{6 n+1} & =(-1)^{n+1} z_{-1}\left(\frac{y_{0}}{x_{-2}-y_{0}}\right)^{3 n+1},
\end{aligned}
$$

$$
\begin{aligned}
x_{6 n+2} & =z_{0}\left(\frac{z_{0}}{y_{-2}}\right)^{3 n+1}, \\
x_{6 n+3} & =y_{-1}\left(\frac{x_{0}}{x_{0}-z_{-2}}\right)^{3 n+2}, \\
y_{6 n-2} & =y_{-2}\left(\frac{z_{0}}{y_{-2}}\right)^{3 n}, \\
y_{6 n-1} & =y_{-1}\left(\frac{x_{0}}{x_{0}-z_{-2}}\right)^{3 n}, \\
z_{6 n+3} & =(-1)^{n} x_{-1}\left(\frac{z_{0}}{y_{-2}-z_{0}}\right)^{3 n+2}, \\
z_{6 n+2} & =x_{-2}\left(\frac{y_{0}}{x_{-2}}\right)^{3 n}, \\
z_{6 n+1} & =(-1)^{n+1} x_{-1}\left(\frac{x_{0}}{y_{-2}-z_{0}}\right)^{3 n+2}, \\
z_{6 n-1} & =(-1)^{n} z_{-1}\left(\frac{x_{0}}{x_{0}-y_{0}}\right)^{3 n+1}, \\
z_{6 n-2} & =z_{-2}\left(\frac{x_{0}}{z_{-2}}\right)^{3 n}, \\
y_{6 n+3} & =(-1)^{n} z_{-1}\left(\frac{x_{0}}{z_{-2}}\right)^{3 n+1}, y_{0} y^{3 n+2},
\end{aligned}
$$

Example 3. Figure 3 represents the behavior of (26) if $x_{-2}=2, x_{-1}=0.81, x_{0}=0.49, y_{-2}=0.3, \quad y_{-1}=-0.09, y_{0}=$ $-0.4, z_{-2}=0.32, z_{-1}=0.12$, and $z_{0}=0.2$.

5. The System: $x_{n+1}==\left(y_{n} z_{n-1} / y_{n}+x_{n-2}\right)$,

$$
\begin{aligned}
& y_{n+1}==\left(z_{n} x_{n-1} / z_{n}-y_{n-2}\right), \\
& \text { and } z_{n+1}==\left(x_{n} y_{n-1} / x_{n}+z_{n-2}\right)
\end{aligned}
$$

We discuss the solutions of the following system: 


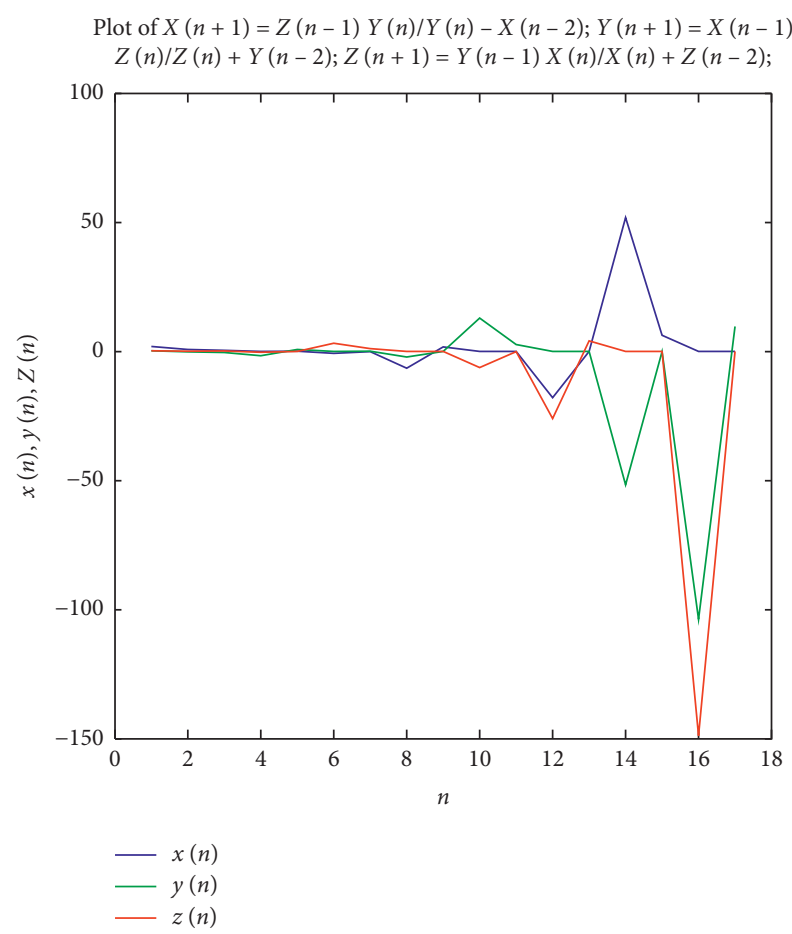

Figure 3: Difference system (26).

$$
\begin{aligned}
& x_{n+1}=\frac{y_{n} z_{n-1}}{y_{n}+x_{n-2}}, \\
& y_{n+1}=\frac{z_{n} x_{n-1}}{z_{n}-y_{n-2}}, \\
& z_{n+1}=\frac{x_{n} y_{n-1}}{x_{n}+z_{n-2}},
\end{aligned}
$$

where $n \in \mathbb{N}_{0}$ and nonnegative initial conditions such that $x_{-2} \neq \pm y_{0}, x_{-2} \neq-2 y_{0}, \quad y_{-2} \neq z_{0}, y_{-2} \neq 2 z_{0}, \quad y_{-2} \neq 3 z_{0}, z_{-2}$ $\neq \pm x_{0}$, and $z_{-2} \neq 2 x_{0}$.

Theorem 4. If $\left\{x_{n}, y_{n}, z_{n}\right\}$ are solutions of (28), then

$$
\begin{aligned}
x_{6 n-2} & =\frac{(-1)^{n} y_{0}^{3 n}}{x_{-2}^{2 n-1}\left(x_{-2}+2 y_{0}\right)^{n}}, \\
x_{6 n-1} & =\frac{x_{-1} z_{0}^{3 n}}{\left(y_{-2}-z_{0}\right)^{2 n}\left(y_{-2}-3 z_{0}\right)^{n}}, \\
x_{6 n} & =\frac{(-1)^{n} x_{0}^{3 n+1}}{z_{-2}^{2 n}\left(2 x_{0}-z_{-2}\right)^{n}}, \\
x_{6 n+1} & =\frac{z_{-1} y_{0}^{3 n+1}}{\left(x_{-2}-y_{0}\right)^{n}\left(x_{-2}+y_{0}\right)^{2 n+1}}, \\
x_{6 n+2} & =\frac{(-1)^{n+1} z_{0}^{3 n+2}}{y_{-2}^{n}\left(y_{-2}-2 z_{0}\right)^{2 n+1}},
\end{aligned}
$$

$$
\begin{aligned}
& x_{6 n+3}=\frac{(-1)^{n} y_{-1} x_{0}^{3 n+2}}{\left(x_{0}-z_{-2}\right)^{2 n+1}\left(x_{0}+z_{-2}\right)^{n+1}} \text {, } \\
& y_{6 n-2}=\frac{(-1)^{n} z_{0}^{3 n}}{y_{-2}^{n-1}\left(y_{-2}-2 z_{0}\right)^{2 n}}, \\
& y_{6 n-1}=\frac{(-1)^{n} y_{-1} x_{0}^{3 n}}{\left(x_{0}-z_{-2}\right)^{2 n}\left(x_{0}+z_{-2}\right)^{n}}, \\
& y_{6 n}=\frac{(-1)^{n} y_{0}^{3 n+1}}{x_{-2}^{2 n}\left(x_{-2}+2 y_{0}\right)^{n}} \\
& y_{6 n+1}=\frac{-x_{-1} z_{0}^{3 n+1}}{\left(y_{-2}-z_{0}\right)^{2 n+1}\left(y_{-2}-3 z_{0}\right)^{n}} \text {, } \\
& y_{6 n+2}=\frac{(-1)^{n+1} x_{0}^{3 n+2}}{z_{-2}^{2 n+1}\left(2 x_{0}-z_{-2}\right)^{n}} \\
& y_{6 n+3}=\frac{-z_{-1} y_{0}^{3 n+2}}{\left(x_{-2}+y_{0}\right)^{2 n+2}\left(x_{-2}-y_{0}\right)^{n}} \text {, } \\
& z_{6 n-2}=\frac{(-1)^{n} x_{0}^{3 n}}{z_{-2}^{2 n-1}\left(2 x_{0}-z_{-2}\right)^{n}} \\
& z_{6 n-1}=\frac{z_{-1} y_{0}^{3 n}}{\left(x_{-2}-y_{0}\right)^{n}\left(x_{-2}+y_{0}\right)^{2 n}}, \\
& z_{6 n}=\frac{(-1)^{n} z_{0}^{3 n+1}}{y_{-2}^{n}\left(y_{-2}-2 z_{0}\right)^{2 n}} \\
& z_{6 n+1}=\frac{(-1)^{n} y_{-1} x_{0}^{3 n+1}}{\left(x_{0}-z_{-2}\right)^{2 n}\left(x_{0}+z_{-2}\right)^{n+1}}, \\
& z_{6 n+2}=\frac{(-1)^{n} y_{0}^{3 n+2}}{x_{-2}^{2 n}\left(x_{-2}+2 y_{0}\right)^{n+1}}, \\
& z_{6 n+3}=\frac{x_{-1} z_{0}^{3 n+2}}{\left(y_{-2}-3 z_{0}\right)^{n+1}\left(y_{-2}-z_{0}\right)^{2 n+1}} .
\end{aligned}
$$

Example 4. Figure 4 represents the dynamics of (28) with $x_{-2}=0.8, x_{-1}=0.25, x_{0}=-0.31, y_{-2}=0.2$, $y_{-1}=0.7, y_{0}=-0.13, z_{-2}=0.4, z_{-1}=0.9$, and $z_{0}=0.71$.

6. The System: $x_{n+1}==\left(y_{n} z_{n-1} / y_{n}-x_{n-2}\right), y_{n+1}$ $==\left(z_{n} x_{n-1} / z_{n}+y_{n-2}\right)$, and $z_{n+1}==$
$\left(x_{n} y_{n-1} / x_{n}+z_{n-2}\right)$ We will obtain the solutions of the following system: 


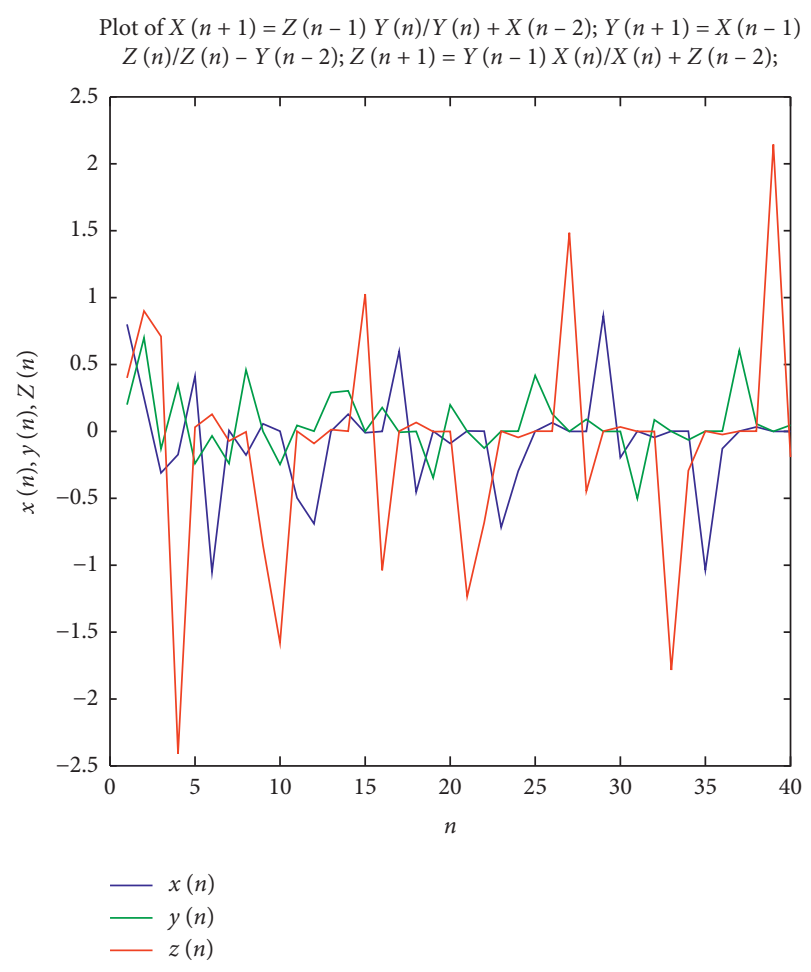

FIgURE 4: Difference system (28).

$$
\begin{aligned}
& x_{n+1}=\frac{y_{n} z_{n-1}}{y_{n}-x_{n-2}}, \\
& y_{n+1}=\frac{z_{n} x_{n-1}}{z_{n}+y_{n-2}}, \\
& z_{n+1}=\frac{x_{n} y_{n-1}}{x_{n}+z_{n-2}},
\end{aligned}
$$

where $n \in \mathbb{N}_{0}$ and nonzero initial conditions such that $x_{-2} \neq y_{0}, x_{-2} \neq 2 y_{0}, x_{-2} \neq 3 y_{0}, \quad y_{-2} \neq \pm z_{0}, y_{-2} \neq 2 z_{0}, z_{-2} \neq$ $\pm x_{0}$, and $z_{-2} \neq-2 x_{0}$.

Theorem 5. If $\left\{x_{n}, y_{n}, z_{n}\right\}$ are solutions of (30), then

$$
\begin{aligned}
x_{6 n-2} & =\frac{(-1)^{n} y_{0}^{3 n}}{x_{-2}^{n-1}\left(x_{-2}-2 y_{0}\right)^{2 n}}, \\
x_{6 n-1} & =\frac{(-1)^{n} x_{-1} z_{0}^{3 n}}{\left(y_{-2}+z_{0}\right)^{n}\left(y_{-2}-z_{0}\right)^{2 n}}, \\
x_{6 n} & =\frac{(-1)^{n} x_{0}^{3 n+1}}{z_{-2}^{2 n}\left(z_{-2}+2 x_{0}\right)^{n}},
\end{aligned}
$$

$$
\begin{aligned}
& x_{6 n+1}=\frac{-z_{-1} y_{0}^{3 n+1}}{\left(x_{-2}-3 y_{0}\right)^{n}\left(x_{-2}-y_{0}\right)^{2 n+1}} \text {, } \\
& x_{6 n+2}=\frac{-z_{0}^{3 n+2}}{y_{-2}^{2 n+1}\left(y_{-2}-2 z_{0}\right)^{n}} \text {, } \\
& x_{6 n+3}=\frac{(-1)^{n+1} y_{-1} x_{0}^{3 n+2}}{\left(x_{0}-z_{-2}\right)^{n}\left(x_{0}+z_{-2}\right)^{2 n+2}} \text {, } \\
& y_{6 n-2}=\frac{z_{0}^{3 n}}{y_{-2}^{2 n-1}\left(y_{-2}-2 z_{0}\right)^{n}} \text {, } \\
& y_{6 n-1}=\frac{(-1)^{n} y_{-1} x_{0}^{3 n}}{\left(x_{0}-z_{-2}\right)^{n}\left(x_{0}+z_{-2}\right)^{2 n}} \text {, } \\
& y_{6 n}=\frac{(-1)^{n} y_{0}^{3 n+1}}{x_{-2}^{n}\left(x_{-2}-2 y_{0}\right)^{2 n}} \text {, } \\
& y_{6 n+1}=\frac{(-1)^{n} x_{-1} z_{0}^{3 n+1}}{\left(y_{-2}-z_{0}\right)^{2 n}\left(y_{-2}+z_{0}\right)^{n+1}} \text {, } \\
& y_{6 n+2}=\frac{(-1)^{n} x_{0}^{3 n+2}}{z_{-2}^{2 n}\left(z_{-2}+2 x_{0}\right)^{n+1}} \text {, } \\
& y_{6 n+3}=\frac{z_{-1} y_{0}^{3 n+2}}{\left(x_{-2}-3 y_{0}\right)^{n+1}\left(x_{-2}-y_{0}\right)^{2 n+1}} \text {, } \\
& z_{6 n-2}=\frac{(-1)^{n} x_{0}^{3 n}}{z_{-2}^{2 n-1}\left(z_{-2}+2 x_{0}\right)^{n}} \\
& z_{6 n-1}=\frac{z_{-1} y_{0}^{3 n}}{\left(x_{-2}-y_{0}\right)^{2 n}\left(x_{-2}-3 y_{0}\right)^{n}}, \\
& z_{6 n}=\frac{z_{0}^{3 n+1}}{y_{-2}^{2 n}\left(y_{-2}-2 z_{0}\right)^{n}} \\
& z_{6 n+1}=\frac{(-1)^{n} y_{-1} x_{0}^{3 n+1}}{\left(x_{0}-z_{-2}\right)^{n}\left(x_{0}+z_{-2}\right)^{2 n+1}} \\
& z_{6 n+2}=\frac{(-1)^{n+1} y_{0}^{3 n+2}}{x_{-2}^{n}\left(x_{-2}-2 y_{0}\right)^{2 n+1}} \text {, } \\
& z_{6 n+3}=\frac{(-1)^{n+1} x_{-1} z_{0}^{3 n+2}}{\left(y_{-2}-z_{0}\right)^{2 n+1}\left(y_{-2}+z_{0}\right)^{n+1}} .
\end{aligned}
$$

Example 5. Figure 5 represents the dynamics of (30) with $x_{-2}=-0.2, x_{-1}=0.05, x_{0}=-0.39, y_{-2}=2, \quad y_{-1}=1.7, y_{0}=$ $-0.3, z_{-2}=1.1, z_{-1}=8$, and $z_{0}=-0.16$. 


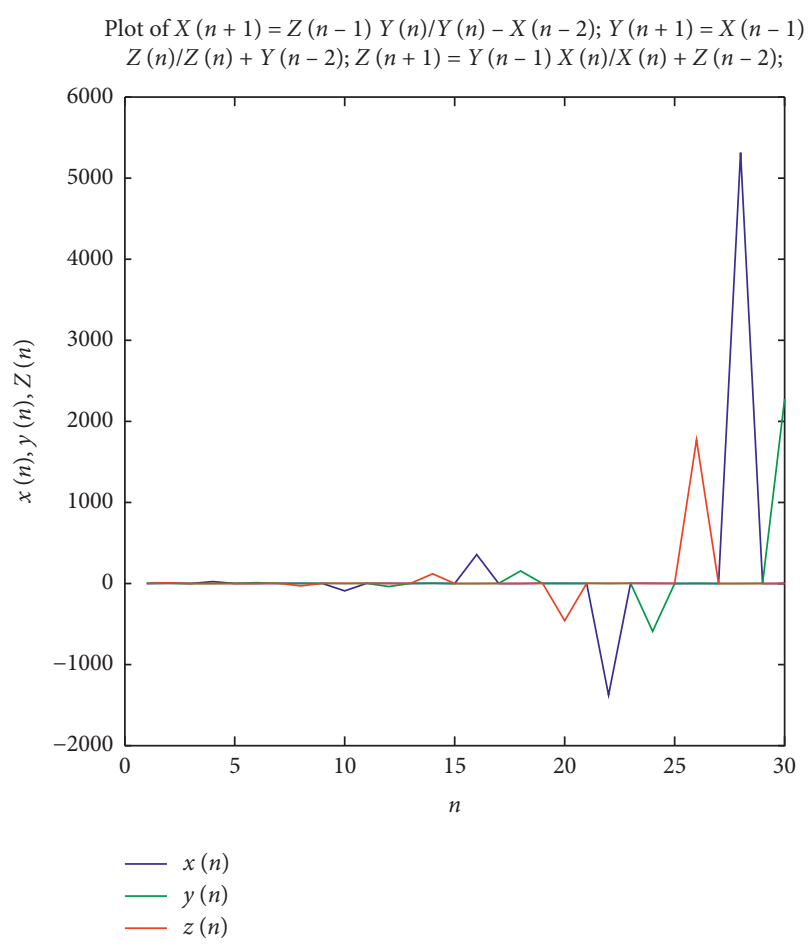

Figure 5: Difference system (30).

\section{Conclusion}

This paper discussed boundedness and the form expressions of the solutions of some special cases of difference equation systems $x_{n+1}=\left(y_{n} z_{n-1} / y_{n} \pm x_{n-2}\right), y_{n+1}=\left(z_{n} x_{n-1} /\right.$ $\left.z_{n} \pm y_{n-2}\right)$, and $z_{n+} 1=\left(x_{n} y_{n-1} / x_{n} \pm z_{n-2}\right)$, after the introduction in Section 1, we investigated in Section 2, the boundedness behavior of equation (10), and we have obtained the solutions of this system. In Section 3, we obtained the form of the solutions of the system $x_{n+1}=\left(y_{n} z_{n-1} / y_{n}+\right.$ $\left.x_{n-2}\right), \quad y_{n+1}=\left(z_{n} x_{n-1} / z_{n}+y_{n-2}\right)$, and $z_{n+1}=\left(x_{n} y_{n-1} / x_{n}\right.$ $\left.-z_{n-2}\right)$. Also, in Section 4, we gave the solution form of the system $x_{n+1}=\left(y_{n} z_{n-1} / y_{n}-x_{n-2}\right), y_{n+1}=\left(z_{n} x_{n-1} / z_{n}-y_{n-2}\right)$, and $z_{n+1}=\left(x_{n} y_{n-1} / x_{n}-z_{n-2}\right)$. In Section 5, we dealt with the solution of system (28). Finally, in Section 6, we have the expressions of the form of the solutions of the system $x_{n+1}=$ $\left(y_{n} z_{n-1} / y_{n}-x_{n-2}\right), y_{n+1}=\left(z_{n} x_{n-1} / z_{n}+y_{n-2}\right)$, and $z_{n+1}=$ $\left(x_{n} y_{n-1} / x_{n}+z_{n-2}\right)$, and in each case, we presented some numerical examples of each case and drew it by using MATLAB.

\section{Data Availability}

All the data utilized in this article have been included, and the sources were it was adopted were cited accordingly.

\section{Conflicts of Interest}

The authors declare that they have no conflicts of interest regarding the publication of this paper.

\section{Acknowledgments}

The work of H. S. Alayachi and M. S. M. Noorani was financially supported by the UKM (grant number DIP-2017011) and Ministry of Education Malaysia (grant number FRGS/1/2017/STG06/UKM/01/1), while A. Q. Khan's research was partially supported by the Higher Education Commission of Pakistan.

\section{References}

[1] R. P. Agarwal, Difference Equations and Inequalities, Marcel Dekker, New York, NY, USA, 1st edition, 1992.

[2] N. Battaloglu, C. Cinar, and I. Yalçınkaya, "The dynamics of the difference equation," ARS Combinatoria, vol. 97, pp. 281-288, 2010.

[3] C. Cinar, I. Yalcinkaya, and R. Karatas, "On the positive solutions of the difference equation system $x_{n+1}=m / y_{n}$, $y_{n+1}=p y_{n} \% / x_{n-1} y_{n-1}$," Journal of Mathematics and Computer Science, vol. 18, pp. 135-136, 2005.

[4] C. Cinar and I. Yalçinkaya, "On the positive solutions of the difference equation system $x_{n+1}=1 / z_{n}, y_{n+1}=y_{n} / x_{n-1} \% y_{n-1}$, $z_{n+1}=1 / x_{n-1}$," Journal of Mathematics and Computer Science, vol. 18, pp. 91-93, 2005.

[5] S. E. Das and M. Bayram, "On a system of rational difference equations," World Applied Sciences Journal, vol. 10, no. 11, pp. 1306-1312, 2010.

[6] Q. Din, M. Qureshi, and A. Q. Khan, "Dynamics of a fourthorder system of rational difference equations," Advances in Difference Equations, vol. 2012, no. 1, p. 215, 2012.

[7] E. M. Elabbasy, H. El-Metwally, and E. M. Elsayed, "On the solutions of a class of difference equations systems," Demonstratio Mathematica, vol. 41, no. 1, pp. 109-122, 2008.

[8] E. M. Elsayed and H. S. Gafel, "The behavior and closed form of the solutions of some difference equations," Journal of Computational Analysis and Applications, vol. 27, no. 5, pp. 849-863, 2019.

[9] E. M. Elsayed, "Solutions of rational difference systems of order two," Mathematical and Computer Modelling, vol. 55, no. 3-4, pp. 378-384, 2012.

[10] E. A. Grove, G. Ladas, L. C. McGrath, and C. T. Teixeira, "Existence and behavior of solutions of a rational system," Communications on Applied Nonlinear Analysis, vol. 8, pp. 1-25, 2001.

[11] L. Keying, Z. Zhongjian, L. Xiaorui, and L. Peng, "More on three-dimensional systems of rational difference equations," Discrete Dynamics in Nature and Society, vol. 2011, Article ID 178483, 9 pages, 2011.

[12] A. S. Kurbanli, C. Cinar, and I. Yalçınkaya, "On the behavior of positive solutions of the system of rational difference equations," Mathematical and Computer Modelling, vol. 53, pp. 1261-1267, 2011.

[13] A. S. Kurbanli, "On the behavior of solutions of the system of rational difference equations," Advances in Difference Equations, vol. 2011, no. 1, p. 40, 2011.

[14] A. S. Kurbanli, "On the behavior of solutions of the system of rational difference equations: $x_{n+1}=x_{n-1} / x_{n-1} y_{n}-1, y_{n+1}$ $\%=y_{n-1} / y_{n-1} x_{n}-1, z_{n+1}=z_{n-1} / z_{n-1} y_{n}-1$, ' Discrete Dynamics in Nature and Society, vol. 2011, Article ID 932362, 12 pages, 2011. 
[15] A. Kurbanli, C. Cinar, and M. Erdoğan, "On the behavior of solutions of the system of rational difference equations $x_{n+1} \%=\left(x_{n-1} / x_{n-1} y_{n}-1\right), y_{n+1}=\left(y_{n-1} / y_{n-1} x_{n} \%-1\right), z_{n+1}$ $=\left(x_{n} / z_{n-1} y_{n}\right)$," Applied Mathematics, vol. 2, pp. 1031-1038, 2011.

[16] A. Y. Özban, "On the system of rational difference equations $x_{n+1}=x_{-2} / y_{n-3}, y_{n+1}=x_{-1} y_{n-3} / x_{n-q} y_{n-q}$," Applied Mathematics and Computation, vol. 188, no. 1, pp. 833-837, 2007.

[17] N. Touafek and E. M. Elsayed, "On the solutions of systems of rational difference equations," Mathematical and Computer Modelling, vol. 55, no. 7-8, pp. 1987-1997, 2012.

[18] I. Yalcinkaya, "On the global asymptotic stability of a secondorder system of difference equations," Discrete Dynamics in Nature and Society, vol. 2008, Article ID 860152, 12 pages, 2008.

[19] I. Yalçınkaya, "On the global asymptotic behavior of a system of two nonlinear difference equations," ARS Combinatoria, vol. 95, pp. 151-159, 2010.

[20] I. Yalcinkaya and C. Cinar, "Global asymptotic stability of two nonlinear difference equations," Fasciculi Mathematici, vol. 43, pp. 171-180, 2010.

[21] I. Yalcinkaya, C. Cinar, and M. Atalay, "On the solutions of systems of difference equations," Advances in Difference Equations, vol. 2008, Article ID 143943, 9 pages, 2008.

[22] X. Yang, Y. Liu, and S. Bai, "On the system of high order rational difference equations $x_{n}=x_{-2} / y_{n-p}, y_{n}=x_{-1} y_{n-p} /$ $x_{n-q} y_{n-q}$," Applied Mathematics and Computation, vol. 171, no. 2, pp. 853-856, 2005.

[23] E. M. E. Zayed and M. A. El-Moneam, "On the rational recursive sequence $x_{n+1}=a x_{n}-\left(b x_{n} / c x_{n}-d x_{n-k}\right)$," Communications on Applied Nonlinear Analysis, vol. 15, pp. 47-57, 2008.

[24] E. M. E. Zayed and M. A. El-Moneam, "On the global attractivity of two nonlinear difference equations," Journal of Mathematical Sciences, vol. 177, no. 3, pp. 487-499, 2011.

[25] E. M. E. Zayed and M. A. El-Moneam, "On the global asymptotic stability for a rational recursive sequence," Iranian Journal of Science and Technology (IJST Transaction A-Science), vol. A4, pp. 333-339, 2011.

[26] H. Sedaghat, Nonlinear Difference Equations Theory with Applications to Social Science Models, Vol. 15, Springer Science \& Business Media, Berlin, Germany, 2003.

[27] V. Kocić and G. Ladas, Global Behavior of Nonlinear Difference Equations of Higher Order with Applications, Kluwer Academic Publishers, Dordrecht, Netherlands, 1993.

[28] M. R. S. Kulenović and G. Ladas, Dynamics of Second Order Rational Difference Equations, Chapman \& Hall/CRC, Boca Raton, FL, USA, 2001.

[29] M. Gumus, "The global asymptotic stability of a system of difference equations," Journal of Difference Equations and Applications, vol. 24, no. 6, pp. 976-991, 2018.

[30] M. Pituk, "More on poincaré's and perron's theorems for difference equations*," Journal of Difference Equations and Applications, vol. 8, no. 3, pp. 201-216, 2002.

[31] M. Gumus and R. Abo-Zeid, "An explicit formula and forbidden set for a higher order difference equation," Journal of Applied Mathematics and Computing, vol. 63, pp. 133-142, 2020.

[32] H. S. Alayachi, A. Q. Khan, M. S. M. Noorani, and A. Khaliq, "Displaying the structure of the solutions for some fifth-order systems of recursive equations," Mathematical Problems in Engineering, vol. 2021, Article ID 6682009, 2021.

[33] H. S. Alayachi, M. S. M. Noorani, A. Q. Khan, and M. B. Almatrafi, "Analytic solutions and stability of sixth order difference equations," Mathematical Problems in Engineering, vol. 2020, Article ID 1230979, 2020.

[34] H. S. Alayachi, M. S. M. Noorani, M. S. M. Noorani, and E. M. Elsayed, "Qualitative analysis of a fourth order difference equation," Journal of Applied Analysis \& Computation, vol. 10, no. 4, pp. 1343-1354, 2020.

[35] A. Khaliq, H. S. Alayachi, M. S. M. Noorani, and A. Q. Khan, "On stability analysis of higher-order rational difference equation," Discrete Dynamics in Nature and Society, vol. 2020, Article ID 3094185, 2020.

[36] E. M. Elsayed, M. M. El-Dessoky, and A. Alotaibi, "On the solutions of a general system of difference equations," Discrete Dynamics in Nature and Society, vol. 2012, Article ID 892571, 12 pages, 2012.

[37] Y. Zhang, X. Yang, G. M. Megson, and D. J. Evans, "On the system of rational difference equations," Applied Mathematics and Computation, vol. 176, no. 2, pp. 403-408, 2006.

[38] O. Zkan and A. S. Kurbanli, "On a system of difference equation," Discrete Dynamics in Nature and Society, vol. 2013, Article ID 970316, 7 pages, 2013. 